\title{
MODELO DE GESTIÓN DE RIESGO DE LA CADENA DE SUMINISTRO COMO ELEMENTO DIFERENCIADOR
}

\section{SUPPLY CHAIN RISK MANAGEMENT MODEL AS A DIFFERENTIATING ELEMENT}

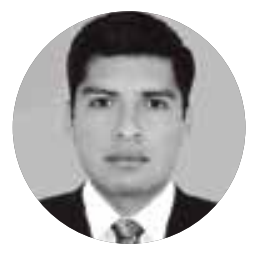

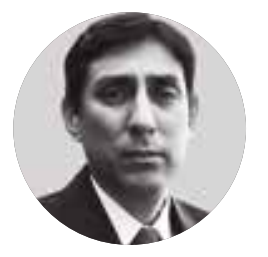

Alex Antonio Izquierdo Requejo

Código ORCID

https://orcid.org/ 0000-0002-2433-4166

Igeneceatecto escia natet, cum sum nume volo tem et quas non re iuntinulla ipident, consequam, sitiorrovid quis cumquib eritiuscias arum simenecum est, corpos

\section{RESUMEN}

Actualmente, las complejidades de la cadena de suministro imponen enormes desafíos en las organizaciones. Esto ha intensificado la necesidad y búsqueda constante de una gestión resiliente. Los riesgos que la amenazan surgen de forma inesperada y agresiva en cualquier punto de la cadena logística y su impacto podría no ser siempre superado favorablemente por las empresas. Asimismo, los responsables deben administrar diversos factores de contingencia en entornos cada vez más competitivos. Esto ha significado hasta hoy en día ubicar métodos de cómo blindar las probables disrupciones en diversos escenarios. No obstante, la gestión integral de riesgos puede ser un enfoque eficaz para hacer frente a estas incertidumbres. En tal sentido, la información desarrollada intenta ser de utilidad a diversas organizaciones y sobre todo a sus gerentes a cargo de la gestión, de modo que tomen decisiones basadas en la experiencia compartida y reflexiva, encaminada a la competitividad empresarial.

Palabras clave: Cadena de suministro, Gestión de riesgo, Procesos, Identificación de riesgo 


\section{ABSTRACT}

Today, the complexities of the supply chain pose enormous challenges for organizations. This has intensified the need and constant search for resilient management. The risks that threaten it arise unexpectedly and aggressively at any point in the logistics chain and their impact may not always be favorably outweighed by companies. Likewise, those responsible must manage various contingency factors in increasingly competitive environments, this has meant until today the establishment of methods of how to shield the probable disruptions in various scenarios. However, comprehensive risk management can be an effective approach to address these uncertainties. In this sense, the information developed aims to be useful to various organizations and especially their managers in charge of management, so that they make decisions based on shared and reflective experience, aimed at business competitiveness.

Keywords: Supply chain, Risk management, Processes, Risk identification.

\section{PLANTEAMIENTO DEL PROBLEMA}

Diariamente en el mundo, cada vez más globalizado, suceden un incontable número de acontecimientos, donde la relevancia de estos sucesos es proporcional a la capacidad de cada organización y lo que su modelo de negocio apuesta en el mercado competitivo definido por su rubro. Esto hace que sus actividades estén ligadas y expuestas constantemente a factores no solo internos, propios y de dominio de la empresa, sino también a las fuerzas externas que no siempre son posibles de tener bajo control o de las que uno pueda defenderse. A interrogantes, como, por ejemplo, qué tan posible es hacer frente a una eventual baja de un personal eficiente; si es fácil generar un crédito financiero para lograr una inversión; qué tan simple es tener un vehículo de respaldo ante la avería de tu transporte principal; y si es atendible recuperar un mes de bajas ventas probablemente, uno responda que resulta sencillo resolver estos problemas. Sin embargo, ¿qué sucede cuando la magnitud de estas interrogantes se multiplica exponencialmente y las empresas se ubican en el centro de una crisis financiera, una recesión mundial, el desempleo globalizado, la inflación, un golpe de Estado, un derrame de petróleo, una expansión de las tendencias digitales, o sin ir muy lejos, la crisis sanitaria, como la que se vive en el mundo por la pandemia Covid-19? Si revisamos la evidencia, las soluciones de supervivencia de las organizaciones se reducen a un número menor de posibilidades. Igualmente, se sabe que las empresas se preocupan por ganar progresivamente mayor presencia en el mercado y subsistir a todo costo, de tal modo que sus bienes y servicios lleguen a quienes los necesitan, en tiempo oportuno y en una presentación adecuada. Los clientes, del mismo modo, se han tornado más exigentes y poco tolerantes a demoras y atenciones que no estén a la altura de sus expectativas. La relación empresa-cliente es muy sensible y cualquier evidencia o variación desfavorable podría simplemente terminar con este vínculo económico (Butner, 2010). Todo este proceso recibe el nombre de "cadena de suministro" (Purohit \& Kumar, 2013).

La cadena de suministro es una secuencia armónica de procesos, donde su redacción y lectura aparentan ser un tema simple, pero que, contrariamente, no lo es. La materialización y gestión de toda esta red logística, como describimos líneas arriba, no son nada fáciles, por lo mismo que cada sección de esta cadena es propensa a sufrir disrupciones y sin importar donde inicie o se ubique la ruptura, ésta puede ocasionar que las metas de la empresa no se logren, lo que se traduce en costos inesperados, o peor, en pérdidas para las empresas (Mulyati, 2015). La importancia de una buena administración de la cadena de suministro recae en la creación de valor en términos de calidad, coste, velocidad y flexibilidad, entre otros. Además, la gestión integrada de la cadena de suministro mejora la capacidad de diseñar productos más rápidamente y mantener el nivel de abastecimiento requerido por el mercado (Ajmera \& Cook, 2009). Asimismo, nos conduce hacia la reducción de costos y al desabastecimiento oportuno, como al tiempo de entrega dentro de los plazos acordados (Talib, 2011); así como a las mejoras en la eficacia del servicio y la eficiencia en reducir gastos (Richey, 2010). En este sentido, la cadena de suministro proporciona muchos beneficios a una empresa. Sin embargo, el cumplimiento de estas ventajas no es una tarea fácil. Existen diversos riesgos que pueden surgir a lo largo de la cadena, los mismos que pueden ser clasificados en tres amplias categorías: operativas, económicas e imagen corporativa (Kleindorfer \& Saad, 2005). 
La presente investigación tiene como objetivo principal abordar la siguiente pregunta: ¿los modelos de gestión de riesgo de la cadena de suministro representan un elemento diferenciador en las organizaciones? Para ello, revisamos la literatura existente y disponible, como las buenas prácticas que desarrollan diferentes modelos de negocio.

\section{MARCO TEÓRICO}

Para la revisión de la literatura existente se han utilizado diversos recursos bibliográficos en correspondencia a la metodología de investigación descriptiva, particularmente artículos científicos validados y clasificados en el Scimago Journal y Country Rank. En dicha revisión se consultó un total de 60 fuentes de correspondencia al tema planteado, realizando un filtro de relevancia basado en su cuartil, para obtener un grupo de 35 trabajos de investigación de este tipo. Estas fuentes corresponden a diversos autores con publicaciones entre los años 2004 y 2018. La información y datos extraídos de las fuentes, fueron clasificadas en tablas de correspondencia a los criterios: modelos de gestión; procesos para tratamientos de riesgos; identificación de riesgos; importancia de localización; y ventaja competitiva. Igualmente, los principales títulos de estos documentos responden a: gestión de riesgos de la cadena de suministro; valor de las estrategias de mitigación y contingencia para gestionar los riesgos de interrupción de la cadena de suministro; estrategias de gestión de riesgos de la cadena de suministro global; mejora del rendimiento de la cadena de suministro y gestión del riesgo ante la incertidumbre relacionada con el clima; evaluación y gestión de riesgos mediante la cadena de suministro; proceso de gestión de riesgos; evaluación de riesgos de la cadena de suministro; entendiendo los riesgos técnicos desde las perspectivas de compañías de la cadena de suministro; identificación de riesgos y mitigación de interrupciones en la cadena de suministro automotriz; redes de acción estratégica: un estudio sobre la gestión del riesgo de la cadena de suministro en las industrias manufactureras; y gestión de riesgos cibernéticos e informáticos en cadenas de suministro: perspectivas de un análisis exploratorio, entre otros títulos de igual importancia para esta investigación.

Para lograr entender los diversos criterios que se desarrollan en la presente investigación, es necesario puntualizar algunos conceptos de mayor relevancia:

\subsection{Gestión administrativa}

Anzola (2002) menciona que "gestión administrativa consiste en todas las actividades que se emprenden para coordinar el esfuerzo de un grupo, es decir, la manera en la cual se tratan de alcanzar las metas u objetivos con ayuda de las personas y las cosas mediante el desempeño de ciertas labores esenciales como son la planeación, la organización, la dirección y el control". Es por ello que el presente análisis afirma que la gestión administrativa es una de las actividades más importantes dentro de los diversos modelos de negocio, ya que, mediante ésta, en consistencia con la visión empresarial, se puede obtener el logro de la misión, y el cumplimiento de los objetivos, a través de la unión de recursos, capacidades y esfuerzos coordinados de toda la fuerza de trabajo que labora dentro de la misma. Toda organización persigue un fin, el mismo que se puede alcanzar mediante un proceso ordenado y que esté limpio de actos que vayan en contra de la buena imagen de las entidades.

\subsection{Riesgo}

La definición de riesgo, por lo general, se refiere a eventos inciertos que pueden afectar el resultado esperado de cada empresa o proyecto, debido a efectos en el costo, el tiempo o en la calidad de los resultados (Hillson, 2004). Por ello, gestionar los riesgos permite el control eficaz y eficiente de los eventos inciertos, permitiendo reducir la probabilidad de ocurrencia, en el caso de que el efecto sea negativo, o de crear una oportunidad de mejora en el caso de que el efecto sea positivo. Si bien es cierto que cada evento disruptivo impacta negativamente a las organizaciones, si se observa el lado positivo, estos eventos ponen en jaque a todos los modelos de negocios y solo los que estén debidamente blindados sabrán reinventarse, ejemplo de ello se observa en los negocios, que debido a la pandemia Covid-19, han tenido que sufrir cambios y adaptarse a un mercado distinto, pero también a nuevos espacios competitivos que han salido a flote debido a este problema de sanidad y salud social.

Los riesgos se pueden clasificar en términos de cuatro tipos de resultado de acuerdo con su probabilidad e impacto, con diferentes características referidas a: frecuencia, gravedad y previsibilidad. Crockford (1986) proporciona una clasificación popular, que caracterizó las 
consecuencias en triviales, pequeñas, medianas y grandes. Como tal, las consecuencias triviales ocurren con una frecuencia muy alta; tienen una gravedad muy baja; y una predictibilidad muy alta. Las pequeñas consecuencias tienen una alta frecuencia; una gravedad baja; y una previsibilidad razonable, pero su ocurrencia es poco frecuente. Las consecuencias medias tienen una frecuencia baja; una gravedad media; y también una previsibilidad razonable, con frecuencia constante. Finalmente, las grandes consecuencias pueden caracterizarse por una frecuencia muy baja; una gravedad alta; y una predictibilidad mínima.

Este marco también se puede aplicar al contexto. Las "pérdidas triviales" son pérdidas que se espera que ocurran en cualquier organización y pueden ser cubiertas por los presupuestos operativos normales (Crockford, 1986). Las "pequeñas pérdidas" pueden presentar pequeños problemas, a menos que su frecuencia sea tan alta que su efecto agregado se acerque al de una sola "pérdida media". Aunque no es preferible, las "pérdidas medianas" no causarían una preocupación seria a la empresa si ocurrieran en intervalos regulares, ya que su costo podría expresarse como un monto anual, y podrían tomarse disposiciones. Una "gran pérdida" presenta el problema más grave: ocurre muy raramente, pero si ocurriera, podría ser catastrófica para la empresa.

\subsection{La incertidumbre}

La incertidumbre es una parte inherente a cualquier objetivo que una organización requiera obtener (Miller, 1992). La incertidumbre es la falta de previsibilidad y puede dar lugar a resultados positivos o negativos (Stein, 2001). La gestión administrativa toma en cuenta la incertidumbre, sobre todo en la etapa inicial de planificación de su modelo de negocio y en cada nuevo paso que decida realizar, debido a que los riesgos pueden afectar contrariamente al desempeño y resultado de sus actividad primarias y secundarias. Si no se gestiona o reduce el impacto del riesgo, éste podría resultar en la desaceleración de la empresa como también su declive de la organización (Royer, 2000).

\subsection{Cadena de suministro}

La cadena de suministro puede definirse como el conjunto de procesos en donde se realizan actividades relacionadas con el flujo y la transformación de bienes, desde la etapa de la materia prima hasta el usuario final. (Ballou, 2004). Las organizaciones a nivel nacional e internacional tienen por objetivo asignar con éxito sus recursos, tales como: materias primas, instalaciones, artículos en proceso, artículos terminados, y puntos de ventas; generándose así un proceso cuyas fases son: [1] la adquisición o suministro de materias primas, [2] desarrollo, proceso y transformación de artículos, y [3] destino o disposición al consumidor final. A este proceso se le conoce como cadena de suministro, la que se conceptualiza como una red de entidades en la cual el material fluye (Lummus, R. 2003).

Estas cadenas de suministro tienden a estar enlazadas entre sus procesos y actividades, tanto principales, como secundarias. Por ello las empresas dependen cada vez más de una compleja red de stakeholders locales y globales, para crear, procesar y hacer entrega de sus bienes y/o servicios en el momento, lugar correcto/oportuno y en la cantidad solicitada. (Quan, Harold, \& Marjolein, 2017). El interés particular detrás de estos esfuerzos es posiblemente el incrementar la probabilidad de éxito y lograr la ventaja competitiva, la permanencia en el mercado, entre otros, ya sea en alguno de sus procesos o, en el mejor escenario, de toda la cadena logística. En respuesta a ello, resulta la necesidad de contar con un enfoque de gestión de riesgo, que intenta estimar y prever la ocurrencia de disrupciones que dificulten, interrumpan o simplemente eviten continuar con la ejecución de su misión, visión y cumplimiento de sus objetivos.

Es relevante señalar que, la gestión de la cadena de suministro se hace evidente desde principios de los 80 s y $90 \mathrm{~s}$ (Oliver \& Webber, 1982). Asimismo, su importancia recae en la dependencia que existe entre los elementos en la cadena de suministro durante todo el proceso, desde el punto de origen del bien o servicio, hasta el punto de consumo de ese bien o servicio (Min \& Mentzer, 2001). Es por lo que la gestión de riesgo de la cadena de suministro identifica una amplia gama de amenazas y riesgos inherentes. Estos riesgos se aplican a las actividades dentro de las estructuras y procedimientos de la empresa, así como externos, es decir, los proveedores y los clientes. En otras palabras, estos riesgos y amenazas pueden afectar a toda la cadena o a un área sensible de esta. Asimismo, los riesgos de las cadenas de suministro pueden afectar a todas las organizaciones de diferente modo e impacto debido a la interconectividad económica que las relaciona.

La gestión de riesgos en la cadena de suministro se ha convertido en uno de los principales temas de investigación con los constantes cambios en el desarrollo globalizado de las organizaciones, considerando las dificultades e im- 
pactos históricos ocurridos en las distintas locaciones donde las empresas desarrollan sus actividades propias del modelo de negocio y mercado en el que compiten. Asimismo, la gestión de riesgos en la cadena de suministro es indispensable, teniendo en cuenta los panoramas económicos e industrializados en el que las empresas intentan operar actualmente (Lavastre, Gunasekaran, \& Spalanzani, 2012).

Es necesario que se tome en cuenta que una empresa con "orientación a la cadena de suministro" reconoce el valor estratégico de administrar actividades operativas y flujos a través de esa. La cadena de suministro requiere la coordinación de un rango amplio de actividades y flujos que se extienden a través de los límites departamentales y de la organización,que incluye: compras; liberación de materiales; transporte para recibir y distribuir; manejo de materiales; almacenamiento y distribución; control y administración de inventarios; planeación de la demanda y abastecimiento; proceso de pedidos; planeación y control de la producción; embarque, proceso y servicio al cliente.

Se debe tomar en cuenta que la planificación en toda la cadena de suministro implica identificar y comunicar los problemas a lo largo de la red logística; establecer estrategias de colaboración; y cooperación y coordinación, enfocadas hacia el tratamiento y control de las fuentes de riesgo, evitando los problemas de asimetrías de información y sus efectos negativos en la gestión de la cadena de suministro y cada una de sus áreas involucradas como también en los grupos de interés que conservan un vínculo de negocio.

\subsection{Resiliencia en la cadena de suministro}

Ponomarov y Holcomb (2009) definen la resiliencia en la cadena de suministros como la capacidad adaptativa de la cadena de prepararse para la ocurrencia de eventos inesperados, y recuperarse de ellos mediante el mantenimiento, la continuidad de las operaciones a un nivel deseado y el control sobre la estructura y el funcionamiento.

\section{METODOLOGÍA DESCRIPTIVA}

\subsection{Criterios y etapas}

Se han establecido criterios o lineamientos basados en la revisión de la literatura respecto a la gestión de riesgo de la cadena de suministro y de mayor relevancia entre las fuentes consultadas. En concordancia a lo explicado, el análisis se divide en cinco bloques o etapas: i) consolidación de un modelo de gestión de la cadena de suministro; ii) identificación de un proceso integral de gestión de riesgo bajo el concepto de resiliencia y prevención; iii) definición de los riesgos de mayor incidencia e importancia en relación con diversos enfoques o modelos de gestión revisados; iv) evidencia de factores en concordancia a su localización de la cadena de suministro; y v) establecimiento e impacto de la gestión de riesgo en la ventaja competitiva de las organizaciones.

\subsection{Diseño exploratorio}

Es pertinente señalar que, para realizar el proceso y búsqueda de la mayor cantidad de información se ha utilizado el enfoque cualitativo - descriptivo. Asimismo, la investigación se desarrolla en base al diseño de teoría fundamentada, es decir, corresponde a una naturaleza sustantiva que tiene como propósito, en este caso particular, la profunda exploración e investigación de los procesos de gestión de riesgo de la cadena de suministro y el impacto a la que está expuesta.

\subsection{Instrumento de recolección}

La recolección de datos fue establecida y construida a través de tablas dinámicas de análisis, explorando entre la información recogida desde las fuentes de investigación, fundamentalmente artículos de revistas de valor pertinente y también material complementario del tipo tesis, en todos los casos, correspondiente a administración y gerencia de empresas, ubicando el material necesario que logre sustentar la teoría descrita en cada elemento y evidenciando el manejo de diversas organizaciones en correspondencia a los objetivos y en el marco del presente trabajo.

\section{ANÁLISIS DE LA INVESTIGACIÓN}

\subsection{Modelo de Gestión}

Como etapa inicial de análisis, se obtuvo los modelos basados en la experiencia, propiamente dicha, de los responsables del área de gestión de riesgo de la cadena de suministro, centrándose en la identificación de riesgos; su clasificación; análisis de escenarios probables de ocurrencia e impacto; planteamiento de planes de contingencia; 
así como la oportuna retroalimentación y constantes sugerencias de mejora, enfocados al manejo de puntos débiles o que necesiten fortalecerse en el desarrollo de la cadena de suministro. El valor de estos diseños organizacionales corresponde a la utilización sistémica de la metodología cualitativa, ya que obtiene una amplia comprensión del cómo, dónde, cuándo, cuanto, por qué, así como todas las interrogantes posibles a considerar antes de la toma de decisiones, logrando tener como base estructural la esencia de las percepciones; la observación en campo; la consolidación de buenas prácticas; el seguimiento directo de los procesos; el alto nivel de involucramiento; y recojo de una constante tormenta de ideas de cada departamento del modelo de negocio, donde los gerentes responsables de la gestión de riesgo son los actores principales; datos que no pueden ser obtenidos fácilmente de otros agentes o áreas comprometidas y de competencia de las organizaciones (Harsh \& Vijaya, 2013).

Seguidamente se describe mayor detalle de cómo estos modelos se caracterizan:

1. El marco teórico de este modelo de gestión, sustenta que la sólida experiencia y el nivel de compromiso de los líderes a cargo de la gestión, son fundamentales para el desarrollo y logro de la misión y la visión de sus representadas, permitiendo basarse, así como proyectarse, en que las fuentes u origen de los riesgos, así como sus conductores, se pueden prevenir, mitigar, ser identificados, evaluados en concepto de relevancia, y priorizados de alguna manera o bajo criterios de impacto propios de cada organización o modelo de negocio, del mismo modo permita hacer frente a las posibles disrupciones al que se exponen diariamente las empresas cada vez más globalizadas (Ritchie \& Brindley, 2007).

2. Proporciona orientación a los gerentes para elegir estrategias de gestión de riesgos basadas en el entorno de la cadena de suministro global (Manuj \& Mentzer, 2008).

3. Considera que la relevancia de la correcta gestión de la cadena de suministro responde a la posibilidad que ofrece a la empresa de alcanzar una ventaja competitiva en el mercado (Cousins \& Spekman, 2003).

4. Plantea que el uso de la experiencia de los gerentes produce la flexibilidad de la cadena de suministro, siendo esta la capacidad de hacer frente a la variación del estado del flujo regular de la cadena, es decir, no evita necesariamente la probabilidad de que algo negativo ocurra, pero sí puede reducir el impacto de riesgo (Chaudhuri, Boer, \& Taran, 2018).

5. Se basa en el estudio cualitativo que incluye entrevistas en profundidad y reuniones de un grupo focal con ejecutivos de alto nivel de la cadena de suministro (Manuj \& Mentzer, 2008).

6. Plantea que la gestión del riesgo de la cadena de suministro es cada vez más importante a medida que aumenta la vulnerabilidad de las cadenas de suministro, asimismo que no importa el tamaño de la empresa u organización, ya que todos dependen de una cadena de suministro y los riesgos a la que están expuestos (Cucchiella \& Gastaldi, 2006).

7. Explora el fenómeno de la gestión de riesgos de forma integral y sistémica de modo que logre plantear las estrategias de gestión de riesgos en las cadenas de suministro globales (Manuj \& Mentzer, 2008).

8. Considera que las habilidades de gestión de riesgos, incluyendo el conocimiento histórico de riesgo y el desarrollo de planes de gestión de riesgos, son requisitos esenciales para el éxito de la gestión de la cadena de suministro en la actualidad y futuro próximo (Giunipero \& Pearcy, 2006).

9. Plantea que la toma de decisión como gestión preventiva de riesgo de la cadena de suministro debe estar directamente relacionada a la incertidumbre y el riesgo de que se trate (Vilko, Ritala, \& Edelmann, 2014). 10. Toma en cuenta que los gerentes deben gestionar muchos riesgos en sus entornos cada vez más competitivos y dinámicos lo que significaba amortiguar las incertidumbres (Giunipero \& Eltantawy, 2004).

11. Recoge de la experiencia de los gerentes que, la incertidumbre significa que pueden ocurrir eventos inesperados, inevitables en su totalidad, pero que se puede cuantificar y por lo tanto ser administrado (Vilko, Ritala, \& Edelmann, 2014).

12. Se considera que las cadenas de suministro están interconectadas y que las empresas dependen cada vez más de una compleja red de socios globales para entregar los productos en el momento y lugar correctos, en la cantidad apropiada, y bajo las presiones de costos pertinentes. Por ello, usa la experiencia de los gerentes para realizar la integración de procesos de negocio, ayudando así a mantener la competitividad y ventaja de las empresas involucradas (Zhu, Krikke, \& Caniëls, 2017). 


\section{REVIEW OF GLOBAL}

MANAGEMENT

FACULTAD

DE NEGOCIOS

DE LA UPC

13. Considera que los efectos de la gestión del riesgo de la cadena de suministro ayudan a las cadenas logísticas a hacer frente a las vulnerabilidades de forma proactiva al respaldar la solidez y la agilidad de los procesos. Se asume que ambas dimensiones influyen en el valor del cliente de la cadena de suministro y en el rendimiento del negocio (Wieland \& Wallenburg, 2012).

14. Este modelo toma en cuenta la experiencia de sus líderes para la innovación en la cadena de suministro, siendo este un aspecto fundamental de la ventaja competitiva de cualquier organización y en última instancia, del éxito. Sin embargo, los riesgos relacionados con la innovación de la cadena de suministro suelen ser catastróficos para las organizaciones. Como tal, la gestión de riesgos haciendo uso de la innovación de la cadena de suministro es clave para cualquier organización que busque obtener una ventaja competitiva. Esta ventaja se obtiene al aumentar la eficiencia, la innovación tecnológica y la innovación de procesos. Estas mejoras pueden aumentar la capacidad operativa, que tiene el efecto de aumentar la capacidad de gestión de riesgos (Kwak, 2018).

15. Para la elaboración de los procesos de gestión se toma en cuenta a la globalización y la externalización, ya que estas han hecho que las empresas sean más vulnerables a la cadena de suministro. En consecuencia, las empresas y sus gerentes líderes deben adoptar diferentes estrategias de gestión de riesgos de la cadena de suministro elaboradas a medida y sin dejar de lado ningún punto probable de disrupción para lograr mitigar los riesgos de la cadena de suministro, logrando blindar los de mayor relevancia para la empresa en un momento determinado (Kumar \& Bhat, 2014).

16. Por último, el modelo propone que la gestión eficaz del riesgo de suministro no consiste solo en adoptar una amplia gama de prácticas sofisticadas de reducción de riesgo. El desafío puede estar más relacionado con encontrar la combinación correcta de prácticas, que se ajuste a los recursos disponibles y sea suficiente para protegerse o blindarse contra los riesgos de suministro (Ellegaard, 2018).

Es pertinente señalar que, previo a la elaboración y consolidación de este diseño de gestión, se toma en cuenta los hallazgos y datos extraídos de la literatura existente, así como de buenas prácticas de las organizaciones que usan dichos modelos, donde se deja constancia que sí es posible identificar métodos que vienen siendo utilizados para superar riesgos complejos e innumerables que caracterizan a todo proceso de la cadena de suministro (Rafisah, 2016). Asimismo, proporcionan una visión y comprensión contemporánea de la gestión de riesgo de la cadena de suministro y se propone un aporte integral sobre toda la metodología que viene siendo utilizada por los distintos modelos de negocio, materia de análisis, y que atienden específicamente a los objetivos del presente tema de investigación, dando lugar a una síntesis de marco conceptual que permita a investigaciones futuras ser guía o insumo de trabajo hacia la maduración de esta disciplina, que es la gestión integral de los riesgos de la cadena de suministro y su impacto que todos los grupos de interés requieren constantemente prevenir o reducir (Fan \& Stevenson, 2018).

\subsection{Proceso de gestión de riesgo}

Como se sabe, el proceso y la gestión administrativa son la agrupación de diversas fases o etapas consecutivas a través de las cuales se efectúa y desarrolla un determinado modelo de negocio. Estas fases deben interrelacionarse; y es así que forman un proceso integral. Igualmente, la correcta administración responde a un ilimitado número de eslabones a través del cual se logran determinados objetivos previamente establecidos (Manrique, M. \& Teves, J. \& Taco, Armando. \& Flores, J. 2018), así como la misión y la visión de las empresas, siendo también correcto señalar que, en ese desarrollo, se debe utilizar racionalmente los recursos humanos, materiales, técnicos y financieros, con el propósito general de disminuir costos; incrementar los márgenes; generar bases sostenibles en el mercado; lograr una marca y su conservación en el tiempo; y, por qué no decirlo, consolidar concretamente los sueños que todo buen emprendedor anhela cumplir.

Asimismo, se ubica la importancia de gestionar eficaz y eficientemente alineados al core del modelo de negocio. En primer lugar, a través de una sólida planificación, que establezca las metas a corto, mediano y largo plazo, así como sus estrategias de acción. En segundo lugar, una oportuna organización, estructurando las actividades y asignación de recursos. En tercer lugar, lograr una dirección sinérgica, que logre desde sus responsables, motivar, comunicar y supervisar las actividades previamente planificadas y organizadas. En cuarto lugar, un cuidadoso control, que permita medir los resultados con el cargo de mejora continua, afianzando puntos débiles y buscando 
oportunidades de los factores sólidos de la empresa.

Por último, pero no menos importante, una consolidada retroalimentación, que recoja todo defecto o fortaleza del proceso con el objetivo de generar valor y sostenibilidad, realizando oportunamente las directivas o disposiciones de corrección o sugerencias de mejora, no solo para el íntegro del proceso, sino también para cada parte de éste, tomando en cuenta que, a pesar de que una actividad haya logrado el éxito, ésta se puede mejorar. A continuación, se expone el esquema de modelo basado en un proceso de cinco fases:

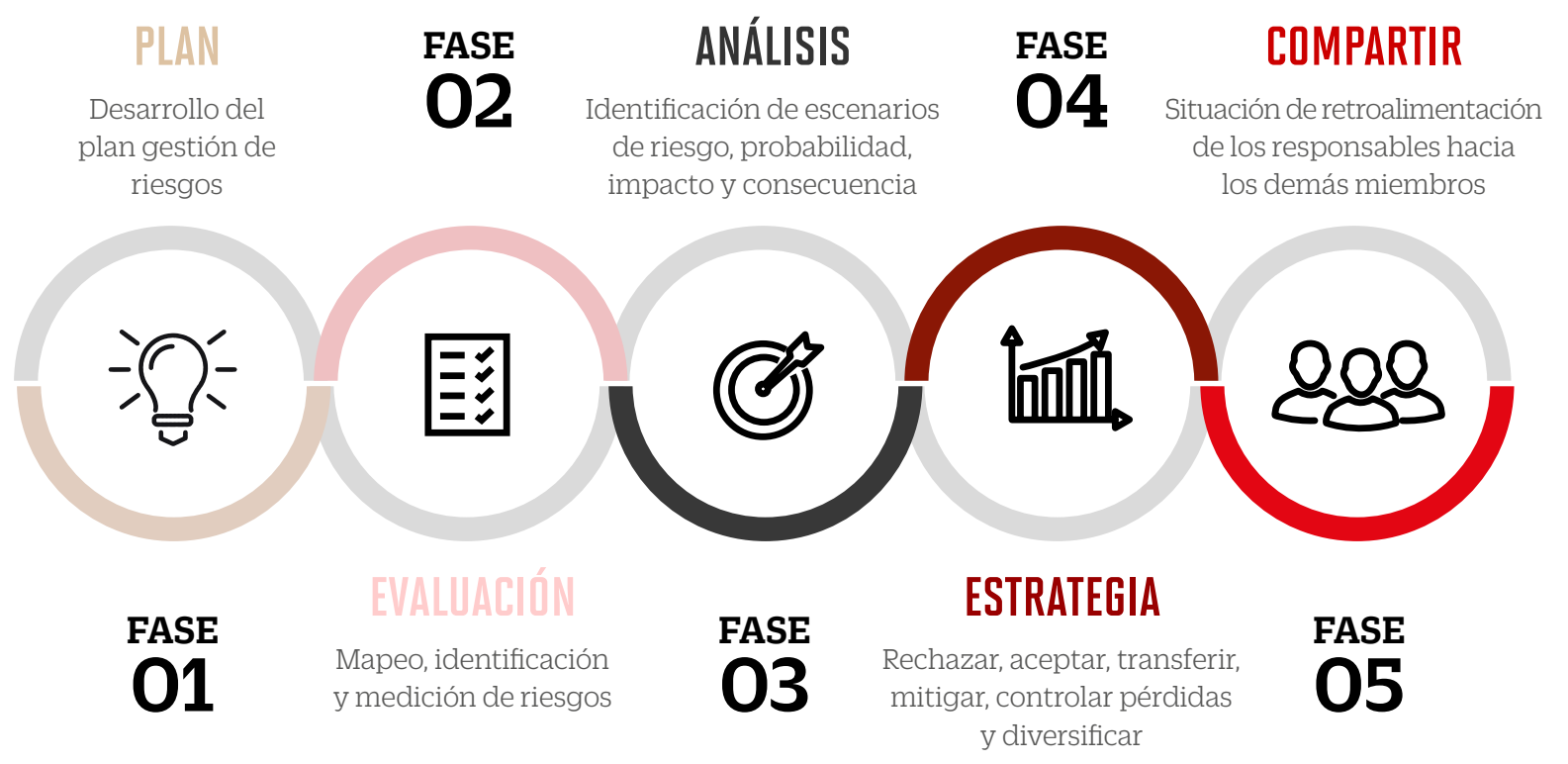

Ilustración 1. Resumen proceso de gestión de riesgos.

\section{Fase 1}

Se desarrolla el plan de gestión de riesgos, enumerando todos los riesgos previsibles; su clasificación y prioridad; y las acciones preventivas y contingentes, junto con un proceso para rastrearlos.

\section{Fase 2}

Se evalúan los riesgos dentro del mapa de procesos de cada organización (LaGoy, 1994), a través del cual se analiza si el riesgo representa un peligro para el medio ambiente o para el ser humano que se vincule con la empresa. Asimismo, la evaluación de riesgos tiene dos componentes. La identificación de riesgos y la medición de riesgos. Igualmente, se toman las siguientes consideraciones para analizar los riesgos: gravedad de la frecuencia del riesgo; predecir el grado de riesgo; medir la velocidad de aparición del riesgo (de alto a bajo, se asume que esto puede ser engañoso); estimación de la cantidad de riesgo; medición de la duración del riesgo; y medir la pérdida potencial y global (cantidad de pérdida en el momento y post evento).

\section{Fase 3}

Se realiza un análisis de riesgos, donde se ejecuta una técnica para identificar los factores que pueden causar el fracaso de una empresa (Haimes, 2011). Asimismo, se indica que el análisis de riesgo toma en cuenta tres factores, como el escenario de riesgo; la probabilidad de escenario; y el vector de daño o las consecuencias resultantes.

\section{Fase 4}

Se toma en cuenta la experiencia de los líderes para el manejo del riesgo, donde hay esencialmente seis formas de manejar estratégicamente cada riesgo según lo descrito por Anderson y Schroder (2010): rechazar, aceptar, transferir, mitigar, controlar pérdidas y diversificar. 


\section{Fase 5}

Las decisiones estratégicas de mitigación. Este concepto es una combinación de medidas basadas en los diversos sucesos relacionados con el riesgo en la cadena de suministro y contención del mismo dentro de todas las áreas de la organización. Se toma en cuenta el riesgo compartido, siendo el resultado de los distintos reportes, informes y mecanismos de retroalimentación a cargo de los responsables de la gestión de riesgo en las empresas. Asimismo, las organizaciones de todo rubro y en cualquier nivel o ubicación de sus procesos, comparten al menos una característica común, a decir, buscar mejorar su desempeño, maximizando su rentabilidad, especialmente en tiempos de crisis. Esto puede significar la diferencia entre obtener una ganancia o pérdida; lograr un servicio al cliente aceptable o inaceptable; o niveles de productividad; o incluso impactando directamente la probabilidad de supervivencia de la empresa (Skipper \& Hanna, 2009).

\subsection{Identificación de riesgo}

Se logró identificar el mayor número de riesgos relevantes dentro de la literatura de investigación explorada, así como su clasificación basada en las prácticas evidenciadas y de mayor incidencia, siendo posible agruparlas en 3 distintos bloques:

\subsubsection{Riesgos Operacionales}

\begin{tabular}{|c|c|c|}
\hline Área & \multicolumn{2}{|c|}{ Descripción del riesgo } \\
\hline \multirow{4}{*}{ Producción } & Avería de maquinaria & Mayor costo del procesamiento de productos \\
\hline & Escasez de operadores con experiencia & Cambios de diseño operativo \\
\hline & Mantener la calidad de los envases & Órdenes de compra manual \\
\hline & Picos de rendimiento durante el proceso & Espacios inadecuados de baja capacidad \\
\hline \multirow{11}{*}{ Aprovisionamiento } & Escasez de materias primas & Selección de socios equivocados \\
\hline & Difícil integración con los proveedores & \multirow{2}{*}{$\begin{array}{l}\text { Fuente de suministro de alta capacidad de } \\
\text { utilización }\end{array}$} \\
\hline & \multirow{2}{*}{$\begin{array}{l}\text { Dificultad de adquisición de material de } \\
\text { proveedores leales }\end{array}$} & \\
\hline & & Inflexibilidad de la fuente de suministro \\
\hline & \multirow{2}{*}{$\begin{array}{l}\text { Conservación de material crudo de } \\
\text { alimentación }\end{array}$} & Baja calidad de productos \\
\hline & & Bancarrota del proveedor \\
\hline & Desabastecimientos de proveedores & Disponibilidad variable de material \\
\hline & Dependencia de proveedores & \multirow{2}{*}{$\begin{array}{l}\text { Inventario extra sin justificación por baja } \\
\text { demanda }\end{array}$} \\
\hline & Incumplimiento de proveedores & \\
\hline & Interrupción de entrega & Múltiples proveedores no fidelizados \\
\hline & Errores de cumplimiento del proveedor & $\begin{array}{l}\text { Cambios frecuentes de proveedores por } \\
\text { incumplimiento }\end{array}$ \\
\hline \multirow{2}{*}{ Desastres naturales } & Desastres naturales, periódicos y a largo plazo & Incertidumbre del clima \\
\hline & Calamidades: inundaciones, tsunamis, otros. & Rutas con dependencia al estado climático \\
\hline
\end{tabular}


REVIEW OF GLOBAL

MANAGEMENT

FACULTAD

DE NEGOCIOS

DE LA UPC

\begin{tabular}{|c|c|c|}
\hline \multirow{4}{*}{ Estructurales } & Incendio en los almacenes o depósitos & Altos costos de capacidad y almacenamiento \\
\hline & Conflicto infraestructuras por distanciamiento & Uso de calefacción o aire acondicionado \\
\hline & $\begin{array}{l}\text { Riesgos de la planta física, vulnerabilidad } \\
\text { estructural }\end{array}$ & $\begin{array}{l}\text { Incorrecta disposición estructural de trabajo } \\
\text { operativo }\end{array}$ \\
\hline & Falta de flexibilidad de capacidad & Demoras en entregas de partes \\
\hline \multirow{8}{*}{ Transporte } & Procedimientos logísticos complejos & Largas distancias \\
\hline & Dificultades de conservación durante recorrido & Despacho heterogéneo \\
\hline & Fallas técnicas & Fragilidad de artículos \\
\hline & Huelgas portuarias & El tiempo de tránsito en relación al tráfico \\
\hline & Retraso en los puertos debido a su capacidad & \multirow{2}{*}{$\begin{array}{l}\text { Capacidad insuficiente o sobrevalorado por } \\
\text { traslado }\end{array}$} \\
\hline & Entregas tardías & \\
\hline & \multirow{2}{*}{$\begin{array}{l}\text { Mayores costos de transporte } \\
\text { por distancia }\end{array}$} & Falta de capacitación de transportistas \\
\hline & & Dependencia de protocolos de seguridad \\
\hline \multirow{10}{*}{ Tecnología } & Desglose de infraestructura de información & Virus informáticos \\
\hline & \multirow{2}{*}{$\begin{array}{l}\text { Falta de integración efectiva del sistema o } \\
\text { extensa }\end{array}$} & Cambios tecnológicos y su obsolescencia \\
\hline & & \multirow{2}{*}{$\begin{array}{l}\text { Equipos de cómputo de rápida } \\
\text { obsolescencia }\end{array}$} \\
\hline & Sistema de redes complejas & \\
\hline & Falta de compatibilidad en plataformas vigentes & Fraude cibernético \\
\hline & Desglose de infraestructura de información & Virus informáticos \\
\hline & \multirow{2}{*}{$\begin{array}{l}\text { Falta de integración efectiva del sistema o } \\
\text { extensa }\end{array}$} & Cambios tecnológicos y su obsolescencia \\
\hline & & \multirow{2}{*}{$\begin{array}{l}\text { Equipos de cómputo de rápida } \\
\text { obsolescencia }\end{array}$} \\
\hline & Sistema de redes complejas & \\
\hline & Falta de compatibilidad en plataformas vigentes & Fraude cibernético \\
\hline \multirow{3}{*}{ Recursos humanos } & Rotación de la gerencia & Bajo disponibilidad de personal \\
\hline & Escaso nivel de experiencia & Cambios organizacionales y funcionales \\
\hline & Prácticas & Baja capacidad de personal existente \\
\hline
\end{tabular}

Tabla 1. Esquema detallado de riesgos operativos. 
4.3.2 Riesgo Financiero

\begin{tabular}{|c|c|c|}
\hline Área & \multicolumn{2}{|c|}{ Descripción del riesgo } \\
\hline \multirow{4}{*}{ Precio de compra } & Volatilidad de precios & Contrato oficial con proveedores \\
\hline & Fluctuación de divisas & Relación cercana no satisfactoria con proveedores \\
\hline & Error en estrategia de precio de compra & Pedidos en base a estimaciones erradas \\
\hline & Acuerdo de bloqueo de precios & Largo pedido de compra \\
\hline \multirow{5}{*}{ Oferta demanda } & Fluctuaciones de la demanda & Pronóstico errado \\
\hline & Predicción precisa & La dependencia de proveedores \\
\hline & Actualizaciones frecuentes & Baja compra por estación del año \\
\hline & Venta y Consumo de riesgo & Pedidos urgentes sin respuesta \\
\hline & $\begin{array}{l}\text { Inestabilidad en la oferta y demanda de los } \\
\text { mercados }\end{array}$ & Estimaciones sin respaldo objetivo \\
\hline \multirow{6}{*}{ Sociopolítico } & Riesgo Gobierno Regulador & Pérdida de control desde el Estado \\
\hline & Inestabilidad regional & Violaciones de propiedad intelectual \\
\hline & Dificultades de comunicación & Riesgo de terrorismo \\
\hline & Regulaciones gubernamentales imprevistas & Inestabilidad laboral \\
\hline & Inestabilidad financiera & Aumento del precio del petróleo \\
\hline & Divisas y los cambios en los tipos de cambio & Cambios en tratados comerciales \\
\hline
\end{tabular}

Tabla 2. Esquema detallado de riesgos financieros.

\subsubsection{Imagen Corporativa}

\begin{tabular}{|l|l|l|l|l|l|l}
\hline \multicolumn{1}{|c|}{ Área } & & Descripción del riesgo \\
\hline & Descuido de imagen organizacional & Imagen que proyectan los usuarios de la marca \\
\hline Reputación & Ofrecer bienes y servicios ilegales & Seguir tendencias de moda antisocial \\
\hline Imagen degradante de clase de bien o servicio & Calidad defectuosa \\
\hline Dañar el medio ambiente & Productos con riesgos para la seguridad \\
\hline Sin acciones de reciclaje o responsabilidad social & Conocimiento inadecuado de la gente \\
\hline Clientes insatisfechos post compra y/o atención & Campañas de marketing mal elaboradas \\
\hline Ofrecer productos que atenten a la & Mala calidad de sus procesos \\
\hline
\end{tabular}

Tabla 3. Esquema detallado de riesgos de imagen corporativa. 


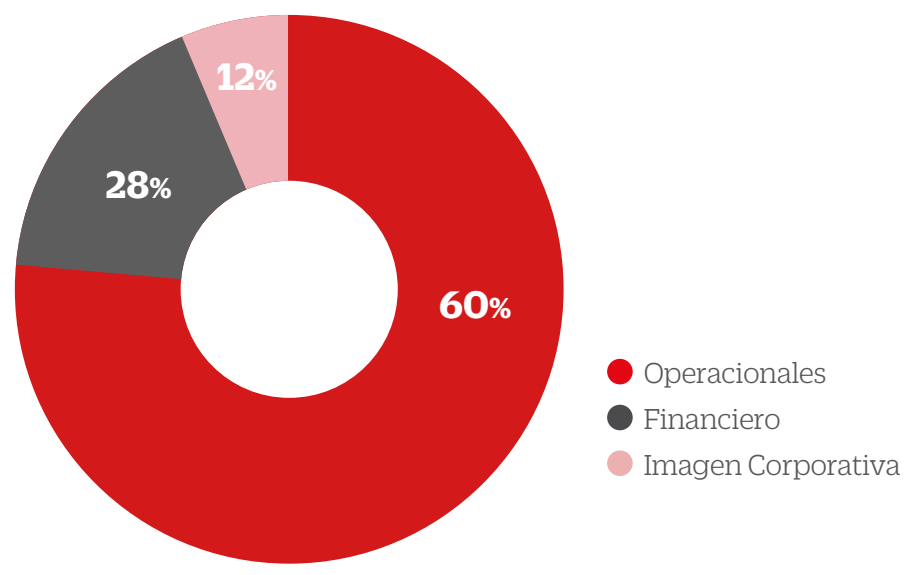

\section{Pirámide de riesgos}

Idenificación y clasificación

\section{RI}

Se conoce como riesgo de imagen corporativa aquel al que se exponen las empresas cuando su prestigio tiene altas probabilidades de ser perjudicados.

\section{RF}

El riesgo financiero se refiere a la probabilidad de ocurrencia de un evento que tenga consecuencias financieras negativas para una organización.

\section{RO}

El riesgo operacional es un tipo de riesgo que puede provocar pérdidas a una empresa debido a errores humanos, procesos internos inadecuados o defectuosos, fallos en los sistemas, entre otros.

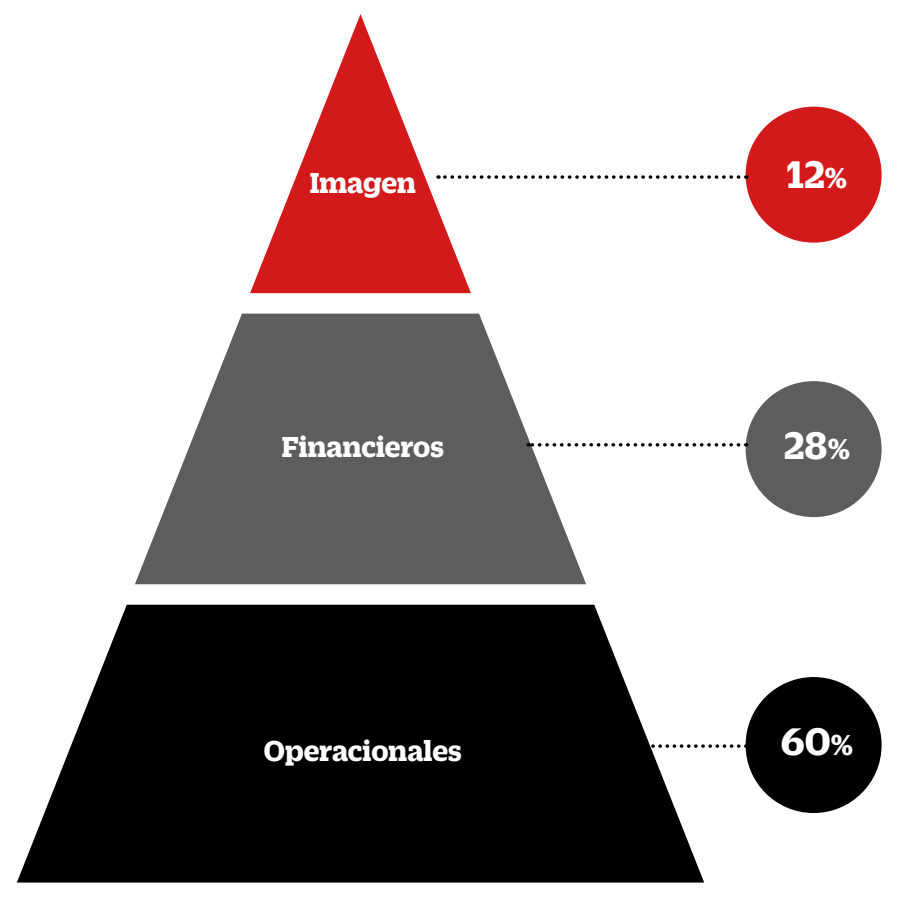




\subsection{Ubicación e impacto de riesgos}

Las decisiones de localización tienen por objetivo el ubicar favorablemente las plantas de producción, centros de distribución, establecimientos de procesamiento, puntos de venta, oficina de gestión, entre otros. Asimismo, la relevancia de estas decisiones hace posible, entre diversos factores, llegar a determinar la asignación del mercado y clientes potenciales a las que se tendría que atender, en concordancia a la proyección de la capacidad de las instalaciones que forman parte de la cadena de suministro y que necesitan ubicarse en algún punto geográfico estratégico, resultado de una decisión responsable y oportuna de parte de los responsables de la gestión del riesgo dentro de las organizaciones. (González, Escorcia, \& Patino, 2017). Asimismo, las decisiones de localización pueden ser las más difíciles que se necesitarían realizar para un eficiente diseño de la cadena logística, debido a la naturaleza estratégica de los diversos modelos de negocio. Sin embargo, es imprescindible hacer un análisis preliminar a profundidad de los riesgos de mayor relevancia y que toda organización adopta apenas pone en marcha su empresa. Además, el decidir donde localizar la cadena de suministro, conlleva también la consideración de costos y presupuestos estructurales a incurrir por sus instalaciones y desarrollo de sus procesos, los que podrían ser elevados o no estar al alcance deseado de los responsables de dichas organizaciones, ya sea por un lento retorno de inversión o por el alto nivel de riesgo de inversión o financiamiento (Daskin, 2002). Es pertinente señalar que, una buena o mala decisión de localización puede marcar la eficiencia y vida del modelo de negocio en correspondencia con la sensibilidad de la cadena de suministro a diversas amenazas de riesgos (Hale \& Moberg, 2003). Por otro lado, los gestores de las cadenas de suministro deben estar en constante exploración de riesgos y su costo-beneficio de localización, para lograr que sus procesos sean robustos y capaces de absorber los impactos negativos, causados por eventos disruptivos, como huracanes, tornados, terremotos, protestas laborales, accidentes, entre otros, que se citan en el punto 5.3.1 de la presente investigación (Gonzales, Escorcia, \& Patiño, 2017).

Es sumamente importante que toda gerencia de riesgo o quienes administren ello, deban tener en cuenta, antes de iniciar un emprendimiento o modelo de negocio, que es crucial saber elegir la localización de dicha organización. Asimismo, se comprenda que una elección apropiada podría impulsar favorablemente el arranque de la empresa, o por el contrario a ello, y sin haber participado un cierto tiempo en el mercado, su destino será un frio y muy profundo fracaso.

El objetivo que se busca establecer en este criterio es el de disponer de una visión detallada de todas las variables posibles y pertinentes dentro del marco del material investigado, que pueden condicionar la viabilidad de localización del establecimiento y ubicación del modelo de negocio, ya sea en el ámbito regional o internacionalmente la producción de determinadas piezas, componentes de procesamiento, entrega o despacho, transporte, entre otros (Guerola, 2009).

A continuación, se exponen algunos factores que toman en cuenta algunas organizaciones de la mano con sus responsables a cargo de la gestión del riesgo de la cadena de suministro.

\subsubsection{Riesgos Operacionales}

\begin{tabular}{|c|c|c|}
\hline Factor & Escenario favorable & Escenario desfavorable \\
\hline $\begin{array}{l}\text { Accesibilidad y } \\
\text { alcance a materias } \\
\text { primas }\end{array}$ & $\begin{array}{l}\text { Contar con insumos y materiales de primer } \\
\text { orden con disponibilidad inmediata }\end{array}$ & $\begin{array}{l}\text { Difícil acercamiento con sus principales } \\
\text { abastecedores de insumos primarios }\end{array}$ \\
\hline $\begin{array}{l}\text { Disponibilidad } \\
\text { de mano de obra } \\
\text { cualificada }\end{array}$ & $\begin{array}{l}\text { Contar con personal del lugar para trabajos } \\
\text { esenciales del modelo de negocio }\end{array}$ & $\begin{array}{l}\text { Necesidad de tener que contratar personal de } \\
\text { otras regiones }\end{array}$ \\
\hline $\begin{array}{l}\text { Acceso a } \\
\text { comunicaciones }\end{array}$ & $\begin{array}{l}\text { Disponer de los servicios básicos de } \\
\text { accesibilidad, tanto físico, como digital }\end{array}$ & $\begin{array}{l}\text { Dificultad de contar con servicios básicos } \\
\text { de comunicación }\end{array}$ \\
\hline
\end{tabular}




\begin{tabular}{|l|l|l|}
$\begin{array}{l}\text { Acceso a } \\
\text { transporte }\end{array}$ & $\begin{array}{l}\text { Lograr alianzas con principales tipos de } \\
\text { transporte, de acuerdo a la necesidad }\end{array}$ & $\begin{array}{l}\text { Baja cobertura de transporte hacia el lugar } \\
\text { donde se ubica sus instalaciones principales }\end{array}$ \\
\hline $\begin{array}{l}\text { Costos favorables } \\
\text { de establecimiento }\end{array}$ & $\begin{array}{l}\text { Precios apropiados y competitivos de renta } \\
\text { o alquiler, así como impuestos }\end{array}$ & $\begin{array}{l}\text { Bajo poder de negociación en cuanto a los } \\
\text { costos por ubicación de instalaciones }\end{array}$ \\
\hline $\begin{array}{l}\text { Proximidad a la } \\
\text { demanda }\end{array}$ & $\begin{array}{l}\text { Lograr ubicar las instalaciones cerca de su } \\
\text { público final objetivo }\end{array}$ & $\begin{array}{l}\text { Lejanía a su público objetivo, incrementando } \\
\text { los costos de acercamiento }\end{array}$ \\
\hline $\begin{array}{l}\text { Ubicación } \\
\text { estratégica de } \\
\text { establecimiento }\end{array}$ & $\begin{array}{l}\text { Disponibilidad favorable de sus principales } \\
\text { servicios }\end{array}$ & $\begin{array}{l}\text { Dificultad de acceder a sus principales } \\
\text { servicios de utilidad primordial }\end{array}$ \\
\hline $\begin{array}{l}\text { Cercanía a } \\
\text { proveedores y } \\
\text { socios estratégicos }\end{array}$ & $\begin{array}{l}\text { Contar con proximidad a su principales } \\
\text { acreedores y negocios de su cadena }\end{array}$ & $\begin{array}{l}\text { Distancia física y de acceso con su principales } \\
\text { y futuros socios estratégicos }\end{array}$ \\
\hline $\begin{array}{l}\text { Proyección } \\
\text { favorable de } \\
\text { crecimiento }\end{array}$ & $\begin{array}{l}\text { Evaluación estadística de crecimiento } \\
\text { favorable en correspondencia al lugar }\end{array}$ & $\begin{array}{l}\text { Estimación sensible de crecimiento oportuno } \\
\text { del negocio en correspondencia al negocio }\end{array}$ \\
\hline $\begin{array}{l}\text { Proximidad } \\
\text { conveniente con } \\
\text { competencia }\end{array}$ & $\begin{array}{l}\text { Distancia favorable con competencia de } \\
\text { productos sustitutos }\end{array}$ & $\begin{array}{l}\text { Acercamiento desfavorable con abastecedores } \\
\text { de productos de amenaza al negocio }\end{array}$ \\
\hline
\end{tabular}

Tabla 4. Esquema de factores y escenarios de localización.

Según diversos autores, una errada decisión puede afectar seriamente la eficiencia general de los resultados de una organización e impactar negativamente en sus procesos y consecuentemente en los clientes (Archie, 2014). No obstante, se logra identificar un proceso para la toma de decisión de localización de la cadena de suministro, que se describe a continuación:

\section{Proceso}

Paso 1

Proceso de competencias internas y externas

\section{Paso 2}

Proceso de relación con los proveedores y clientes

\section{Paso 3}

Proceso de espacio y tiempo

\section{Descripción}

- Análisis interno y visión panorámica de todas la variables y condiciones de ubicación

- Se toma en cuenta el rubro de la organización

- Valora alternativas

- Se revisa el mercado y beneficios de ubicación

- Se identifica a los proveedores potenciales

- Se determina la necesidad y requerimiento que debe afrontar

- Se valora la capacidad de respuesta que requiere de acuerdo con su demanda de sus clientes

- Valora los riesgos logísticos de la cadena de suministro

- Determina los costos de transporte

- Determina los tiempos y plazos que debe cubrir dentro de la cadena de suministro

- Determina las dificultades geográficas

- Relaciones con el gobierno de turno 


\section{REVIEW OF GLOBAL}

\section{MANAGEMENT}

FACULTAD

DE NEGOCIOS

DE LA UPC

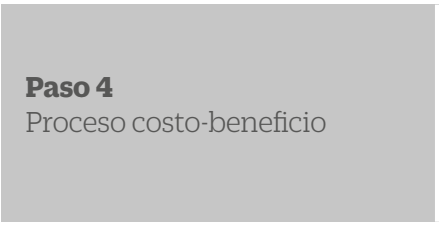

- Identifica oportunidades y beneficios

- Elabora cuadro de análisis comparativo

- Se sustenta, en base a datos estadísticos

- Decide en suma de todas las posibilidades proyectadas y en base al aporte de la

experiencia de sus líderes y gestores

Tabla 5. Esquema del proceso para decisión de localización.

\subsection{La gestión de riesgos como ventaja competitiva}

Se ha explorado diversas fuentes de mayor incidencia, que describen a la gestión de riesgo de la cadena de suministro como factor relevante de la ventaja competitiva, señalando que por intermedio de estos procesos, algunas organizaciones, materia de análisis, señalan haber obtenido un resultado favorable, como también otras que determinan no haber logrado sus objetivos, a pesar del esfuerzo de sus gestiones. Asimismo, en esa dirección y correspondencia, se pretende determinar el efecto e impacto de la utilización de una gestión de riesgo (a costo propio) dentro de las organizaciones y sus directamente responsables de la cadena de suministro, en función y búsqueda de la ventaja competitiva o en su contrapartida, el asumir el riesgo disruptivo en caso que no se cuente con una gestión dedicada del tratamiento de dichos riesgos (Zerón, 2013).

Por un lado, algunos autores como Alexander Correa, Rodrigo Gómez entre otros que se lista en tabla siguiente, se inclinan a favor de que, toda gestión, innovación, acción o proceso propuesto, por lo general requiere de un fuerte apoyo de los principales miembros de una organización o red, para así tener éxito. Es así que es determinante contar con el apoyo de una gestión resiliente de la alta dirección, como también a través de la gestión de riesgo de la cadena de suministro, siendo estos factores importantes porque impactan en los procesos. Es así como, los administradores o gerentes pueden responder mejor a algunos de sus aspectos claves de planificación mediante la mejora constante y blindaje de sus niveles y procesos enfocados a la mitigación de riesgos o planes de contingencia en situaciones de amenaza a la cadena de suministro y a la red que su modelo de negocio pertenece y compite.

Por otro lado, y en contraste a esta teoría, se obtiene que otros autores como Berman Kayis, entre otros que se lista en tabla siguiente, se oponen con su postura, que plantea que una estrategia efectiva de gestión de riesgo de la cadena de suministro es un componente necesario en la estrategia general de la cadena logística de una empresa. Sin embargo, las organizaciones que aceptan planificar y presupuestar costos para atender el riesgo de interrupciones en sus procesos, se exponen también al peligro de una pérdida financiera y de mercado a pesar de sus esfuerzos. Siendo así, las estrategias de gestión de riesgos de interrupciones se basan en acciones de mitigación y/o contingencia, pero no son garantía de éxito de un resultado o escenario favorable. 


\begin{tabular}{|c|c|c|}
\hline Item & Autor(es) & Opinión explorada \\
\hline 1 & Abhijeet Ghadge, Samir Dani y Roy Kalawsky & A favor \\
\hline 2 & Alexander Correa y Rodrigo Gómez & A favor \\
\hline 3 & Andreas Norrman y Ulf Jansson & En contra \\
\hline 4 & Atanu Chaudhuri, Harry Boer y Yariv Taran & A favor \\
\hline 5 & Berman Kayis y Putu Dana Karningsih & En contra \\
\hline 6 & Brian Tomlin & En contra \\
\hline 7 & Luis Alfredo Cabrera Albornoz & A favor \\
\hline 8 & David L. Olson y Desheng Dash Wu & A favor \\
\hline 9 & David Simchi-Levi et al. & A favor \\
\hline 10 & Dong-Wook Kwak y Young-Joon Seoy & A favor \\
\hline 11 & Erjie Ang, Dan A. Iancu y Robert Swinney & A favor \\
\hline 12 & Frank Youhua Chen y Candace Arai Yano & A favor \\
\hline 13 & Guido J.L. Micheli y Enrico Cagno & En contra \\
\hline 14 & Heti Mulyati & A favor \\
\hline 15 & Jason M Riley, Richard Klein, Janis Miller y V. Sridharan & Een contra \\
\hline 16 & Joseph B. Skipper y Joe B. Hanna & Aa favor \\
\hline 17 & Jyri Vilko, Paavo Ritala y Jan Edelmann & En contra \\
\hline 18 & Kristian Rotaru, Carla Wilkin y Andrzej Ceglowski & En contra \\
\hline 19 & F. Lemke y H. L. Petersen & Een contra \\
\hline 20 & Lian Qi, Zuo-Jun Max Shen y Lawrence V. Snyder & A favor \\
\hline 21 & Libiao Bai & A favor \\
\hline 22 & I. Manuj y J. T. Mentzer & En contra \\
\hline 23 & M. Christopher, C. Mena, o. Khan y O. Yurt & A favor \\
\hline 24 & Peter Trkman y Marcos Paulo Valadares & En contra \\
\hline 25 & H. Purohit y V. Kumar & A favor \\
\hline 26 & Mat Radzi Rafisah & A favor \\
\hline 27 & Rao Tummala y Tobias Schoenherr & En contra \\
\hline 28 & Gustavo Rosales & En contra \\
\hline 29 & Sameer Kumar, Katie J. Himes y Collin P. Kritzer & En contra \\
\hline 30 & Uta Jüttner & En contra \\
\hline 31 & Q. Zhu, H. Krikke y M. C. J. Caniëls & En contra \\
\hline
\end{tabular}




\section{REVIEW OF GLOBAL}

MANAGEMENT

FACULTAD

DE NEGOCIOS

DE LA UPC

\section{CONCLUSIONES}

El uso de modelos o guías de gestión de riesgo de la cadena de suministro se considera una base estructural importante para el core del negocio, de modo que se logre un aumento en los beneficios no solo de la empresa, sino también de todos los grupos de interés, dado que las decisiones que se tomen en este primer momento o etapa tendrán un impacto considerable sobre el retorno de la inversión de la cadena. Por tanto, el objetivo de dicho modelo de gestión de riesgo de la cadena de suministros, es el de desarrollar el plan de trabajo, debidamente sustentado en un correcto análisis de línea base de la organización. Es en este punto donde radica la importancia de la experiencia y el compromiso de sus principales gestores de riesgo, haciendo uso de herramientas orientadas a una gestión dinámica y flexible, que logre recopilar de cerca datos estadísticos, conceptos basados en la prevención y respaldo de los puntos más sensibles del modelo de negocio. De este modo, se podrá prever estrategias de mitigación del riesgo y la correcta orientación del presupuesto hacia el blindaje de dichas etapas, siempre constantes en la búsqueda del costo-beneficio, así como la generación de valor y sostenibilidad para la empresa. Un plan de contingencia, adecuadamente preparado, debe detallar una respuesta oportuna y completa a un riesgo específico o a un grupo de riesgos (La Londe, 2005). Diversas opiniones señalan que las organizaciones no siempre pueden ser capaces de predecir o de evitar una interrupción a causa de un riesgo producido en algún punto de la cadena de suministro, pero pueden reducir su exposición mediante la mejora progresiva de sus procesos, implementando herramientas clave de planificación estratégica. Siendo el caso que, si se atienden favorablemente, estas iniciativas estratégicas mejoran la eficiencia de la empresa, como también al grupo de interés relacionado con su red logística, de este modo lograrían responder ante una disrupción, minimizando los impactos negativos del suceso en los niveles generales de rendimiento de la cadena de suministro. (Skipper \& Hanna, 2009)

Actualmente, las cadenas de suministro se exponen a una mayor y creciente probabilidad de experimentar interrupciones en sus procesos, siendo perjudicial para los intereses de las organizaciones. Esto tiende a ser proporcional a su tamaño; a la naturaleza dinámica y compleja de su red logística; así como a la exigente demanda de sus diversos clientes por bienes y/o servicios de calidad y al mejor precio; y las expectativas de crecimiento del modelo de negocio (Blackhurst, 2011). La necesidad de procesos eficaces para el tratamiento del riesgo de la cadena de suministro, resulta indispensable para lograr atender favorablemente la cadena de suministro, sobre todo en el ámbito global, donde las distancias entre los proveedores, mercados, clientes, entre otros, son a menudo de mayor dimensión y riesgo que en las cadenas de suministro local

De la revisión de la literatura, se advierte que existen opiniones divididas respecto a si la gestión de riesgo de la cadena de suministro representa un elemento diferenciador para lograr una ventaja competitiva para las organizaciones que aplican esta metodología. Esta controversia permite explorar razones que sustentan posiciones diversas. Por un lado, un modelo de gestión posibilita a las empresas extender al máximo sus capacidades de mitigación a través de la exploración de las probabilidades de disrupción de sus puntos de mayor vulnerabilidad. Por otro lado, consideran que, a pesar de estos esfuerzos, no siempre se logra tener el control de estos impactos, lo que evidenciaría la ineficacia y costes de dichos esfuerzos. Sin embargo, también se colige que una guía de ruta de trabajo tiende a ser clave para el proceso y desarrollo del ciclo de vida del modelo de negocio y, si bien ninguna estrategia podría predecir a exactitud el impacto del riesgo y como evitarse, sí lograría mitigar su impacto o ubicar a la empresa en una posición de mayor defensa ante diversas disrupciones posibles.

Es posible identificar una perspectiva diferente de cómo entender el concepto de riesgo, su valoración en razón a su impacto, su tratamiento ligado a la prioridad de los procesos dentro de las organizaciones, así como ubicar elementos diferenciadores a través del modelo de gestión que decida ejecutar la empresa. En el presente análisis descriptivo se logra exponer diversos criterios que contribuyen a entender las disrupciones a las que se exponen los diferentes navegantes de un mar tan competitivo como es el mundo de los negocios. Por ello es importante que se continúe con la investigación específica de algunas interrogantes no atendidas en la presente exploración pero que, no por ello, carecen de valor, como, por ejemplo: ¿cuál sería el tratamiento de cada tipo de riesgo expuesto? ¿qué rubros de negocio tienen la mayor incidencia o frecuencia de que sucesos disruptivos 
ocurran? ¿qué tipos de riesgos han resultado en el fracaso de un modelo de negocio? ¿cuáles son las cadenas de suministro más resistentes al impacto de los riesgos? ¿toda gestión de riesgo representa una inversión o un gasto para la empresa? ¿el uso de medios tecnológicos representa la reducción de riesgos o el incremento de éstos? y ¿son los riesgos reputacionales los sensibles, pero a su vez los más difíciles de reparar?

\section{REFERENCIAS}

Abhijeet, G. \&amp; Samir, D. \&amp; Chester, M. \&amp; Kalawsky, A. (2013). A systems approach for modelling supply chain risks. Supply Chain Management, 18(5), 523-538.

Ajmera, A. \&amp; Cook, J. (2009). A Multi-Phase Framework for Supply Chain Integration. S.A.M. Advanced Management Journal, 74(1), 37-47.

Anderson, T. \&amp; Schroder, P. (2010). Strategic risk management practice. New York: Cambridge University Press. United States: Library of Congress Cataloguing in Publication data.

Ang, E. \&amp; Iancu, D. \&amp; Swinney, R. (2017). Disruption Risk and Optimal Sourcing in Multitier Supply Networks. Management Science, 63(8), 2397-2419.

Anzola, S. (2001). Administración de Pequeñas Empresas. McGraw Hill 2a edición.

Archie, L. (2014). Assessing disaster risks in supply chains. Industrial Management \&amp; Data Systems, 114(5), 755-777.

Ballou, H. (2004). Logística: Administración de la cadena de suministro. Pearson Educación.

Blackhurst, J. \&amp;. (2011). An empirically derived framework of global supply resiliency. Journal of Business Logistics, 32(4), 374-391.
Blos, M. \&amp; Quaddus, M. \&amp; Watanabe, K. (2009). Supply chain risk management (SCRM): a case study on the automotive and electronic industries in Brazil. Supply Chain Management: An International Journal, 14(4), 247-252.

Butner, K. (2010). The smarter supply chain of the future. Strategy \& Leadership, 38(1), 22-31.

Cagno, G. (2008). Suministro de gestión del riesgo frente a la selección de proveedores para gestionar la riesgo de suministro en el EPC cadena de suministro. Management Research News, 31(11), 846-866.

Chang, W. \& Ellinger, A. \& Blackhurst, J. (2015). A contextual approach to supply chain risk mitigation. The International Journal of Logistics Management, 26(3), 642-656.

Chaudhuri, A. \& Boer, H. \& Taran, Y. (2018). Supply chain integration, risk management and manufacturing flexibility. International Journal of Operations \& Production Management, 38(3), 690-712.

Chen, F. \& Yano, C. (2010). Improving Supply Chain Performance and Managing Risk Under WeatherRelated Demand Uncertainty. Management Science, 56(8), 1380-1397.

Christopher, M. \& Mena, C. \& Khan, O. \& Yurt, O. (2011). Approaches to managing global sourcing risk. Supply Chain Management: An International Journal, 16(2), 67-81.

Colicchia, C. \& Strozzi, F. (2012). Supply chain risk management: a new methodology for a systematic literature review. Supply Chain Management: An International Journal, 17(4), 403-418.

Correa, A. \& Gómez, R. (2009). Tecnologías de la información y comunicación en la gestión. Revista Avances en Sistemas e Informática, 76(157), 37-48.

Cucchiella, F. \& Gastaldi,M. (2006). Risk management in supply chain: a real option approach. Journal of Manufacturing Technology Management, 17(6), 700-720. 


\section{REVIEW OF GLOBAL}

MANAGEMENT

FACULTAD

DE NEGOCIOS

DE LA UPC

Daskin, M. (2002). An inventory-location model: Formulation, solution algorithm and computational results. Annals of Operations Research, 110(1), 83-106.

Duclos, L. \& Vokurka, R. \& Lummus, R. (2003). A conceptual model of supply chain flexibility. Industrial Management \& Data Systems, 103(6), 446-456.

Ellegaard, C. (2018). Supply risk management in a small company perspective. Supply Chain Management: An International Journal, 13(6), 425-434.

Fan, Y. \& Stevenson, M. (2018). A review of supply chain risk management: definition, theory, and research agenda. International Journal of Physical Distribution \& Logistics Management, 48(3), 205-230.

Finch, P. (2004). Supply chain risk management. Supply Chain Management: An International Journal, 9(2), 183-196.

Gaudenzi, B. \& Borghesi, A. (2006). Managing risks in the supply chain using the AHP method. The International Journal of Logistics Management, 17(1), 114-136.

Ghadge, A. \& Dani, S. \& Chester, M, \& Kalawsky, M. (2013). A systems approach for modelling supply chain risks. Supply Chain Management: An International Journal, 18(5), 523-538.

Giunipero, L. \& Eltantawy, A. (2004). Securing the upstream supply chain: a risk management approach. International Journal of Physical Distribution \& Logistics Management, 34(9), 698-713.

Giunipero, L. \& Pearcy, D. (2006). World Class Purchasing Skills: An Empirical Investigation. Journal of Supply Chain Management, 36(3), 4-13.

González, F. \& Escorcia, J. \& Patino, L. (2017). Optimal and reliable facility location in a supply chain. Revista chilena de ingeniería, 25(4), 4.

Haimes, Y. (2015). Risk modeling, assessment, and management. John Wiley and Sons, 4(1), 1.

Hale, T., \& Moberg, Ch. (2003). Location science research: A review. Annals of Operations Research. Annals of
Operations Research, 123(1), 21-35.

Harsh, P. \& Vijaya, K. (2013). Understanding the Technical Risks from the Perspectives of Dubai Logistics Supply Chain Companies. Asia-Pacific Journal of Management Research and Innovation, 9(3), 291-303.

Hernández, R., Fernández, C., \& Baptista, P. (2014). Metodología de la Investigación. (6a edición). McGraw Hill Interamericana.

Hillson, D. (1997). Project Risk Analysis and Management Guide. EEUU: Association for Project Management, Norwich.

Khan, O., \& Burnes, B. (2007). Risk and supply chain management: creating a research. The International Journal of Logistics Management, 18(2), 197-216.

Kleindorfer, P. (2009). Managing disruption risks in supply chain. Production and Operations Management. Production and Operations Management, 14(1), 53-68.

Kumar, K. \& Bhat, A. (2014). Supply chain risk management dimensions in Indian automobile industry: A cluster analysis approach. Benchmarking: An International Journal, 21(6), 1023-1040.

Kwak, D. \&. (2018). Investigating the relationship between supply chain innovation, risk management capabilities and competitive advantage in global supply chains. International Journal of Operations \& Production Management, 38(1), 2-21.

La Londe, B. (2005). Fiddling while rome burns. Supply Chain Management Review, 9(7), 6.

LaGoy, P. (1994). Risk assessment: Principles and applications for hazardous waste and related sites. New Jersey: Reprint Edition.

Lavastre, O. \& Gunasekaran, A. \& Spalanzani, A. (2012). Supply Chain Risk Management in French Companies. Decision Support Systems, 52(4), 828-838.

Lian, Q. \& Zuo-Jun, M. \& Lawrence, V. (2010). The Effect of Supply Disruptions on Supply Chain Design Decisions. Transportation Science, 44(2), 151-290. 
Libiao, B. \& Chunming, S. \& Yuntao, G. \& Qiang, D. \& Youdan, H. (2018). Quality Risk Evaluation of the Food Supply Chain Using a Fuzzy Comprehensive Evaluation Model and Failure Mode, Effects, and Criticality Analysis. Journal of Food Quality, 2018(1), 1-19.

Lockamy, A. (2014). Assessing disaster risks in supply chains", Industrial Management \& Data Systems. Industrial Management \& Data Systems, 114(5), 755-777.

Manrique, M. \& Teves, J. \& Taco, Armando. \& Flores, J. (2018). Gestión de cadena de suministro: una mirada desde la perspectiva teórica. Revista Venezolana de Gerencia, 24(1), 88.

Manuj, I. \& Mentzer, J. (2008). Global supply chain risk management strategies. International Journal of Physical Distribution \& Logistics Management, 38(3), 192-223.

Mentzer, J. (2001). Defining Supply Chain Management. Journal of Business Logistics, 22(2), 1-25.

Miller, K. (1992). A framework for integrated risk management in international business. Journal of International Business Studies, 23(1), 311-331.

Mulyati, H. (2015). Supply Chain Risk Management Study of the Indonesian Seaweed Industry. Göttingen: Garut.

Norrman, A. \& Jansson, U. (2004). Ericssons proactive supply chain risk management approach after a serious sub supplier accident. International Journal of Physical Distribution \& Logistics Management, 34(5), 434-456.

Oliver, R. \&. (1982). Supply-chain management: logistics catches up with strategy. Journal of Critical Reviews, 1(1), 63-75.

Olson, D. \&. (2010). A review of enterprise risk management in supply chain. Emerald - Kybernetes, 39(5), 694-706.

Olson, D. \&. (2011). Risk management models for supply chain: a scenario analysis of outsourcing to China. Supply Chain Management, 16(6), 401-408.
Parra, S. (2016). Beneficios y ventajas competitivas de la Norma ISO 28000 para la seguridad en la cadena de suministros de empresas nacionales. Administración de la Seguridad.

Petersen, F. \& Lemke y H. (2012). Teaching reputational risk management in the supply chain. Supply Chain Management, 18(4), 413-428.

Ponomarov, S. \& Holcomb, M. (2009). Understanding the Concept of Supply Chain Resilience. The International Journal of Logistics Management, 20(1), 124-143.

Purohit, H. \& Kumar, V. (2013). Supply Chain Risk Evaluation: Understanding the Technical Risks from the Perspectives of Dubai Logistics Supply Chain Companies. Asia-Pacific Journal of Management Research and Innovation, 9(3), 291-303.

Qi, L. \& Shen, Z. \& Snyder, L. (2010). The Effect of Supply Disruptions on Supply Chain Design Decisions. Transportation Science, 44(2), 151-290.

Rafisah, R. \& Intan, S. \& Nazdri, G. (2016). Risk Management in Food Supply Chains by Japanese Food Companies in Malaysia. International Journal of Business Management and Economic Research, 7(6), 778-787.

Rao, S. \& Goldsby, T. (2009). Supply chain risks: a review and typology. The International, 20(1), 97-123.

Richey, R.G. \& Adams, F. \& Dalela, V. (2012). Technology and flexibility: enablers of collaboration and time-based logistics quality. Journal of Business Logistics, 33(1), 34-49.

Riley, M. \& Klein, R. \& Sridharan, J. (2016). How internal integration, information sharing, and training affect supply chain risk management capabilities. International Journal of Physical Distribution \& Logistics Management, 46(10), 953-980.

Rincón, O. (2013). Cuáles son los beneficios de ISO 28000 y BASC en la cadena de Suministros. Administración de la Seguridad y Salud Ocupacional. 


\section{REVIEW OF GLOBAL}

MANAGEMENT

FACULTAD

DE NEGOCIOS

DE LA UPC

Ritchie, B. \& Brindley, C. (2007). Supply chain risk management and performance: A guiding framework. International Journal of Operations \& Production Management, 27(3), 303-322.

Rotaru, K. \&. (2010). Analysis of SCOR's approach to supply chain risk management. International Journal of Operations \& Production Management, 34(10), 1246-1268

Royer, P. (2000). Risk Management: The Undiscovered Dimension of Project Management. Proyect Management Journal, 31(1), 6-13.

Simchi-Levi, D. (2015). Identifying Risks and Mitigating Disruptions in the Automotive Supply Chain. Journal on Applied Analytics, 45(5), 375-390.

Skipper, J. \& Hanna, J. (2009). Minimizing supply chain disruption risk through enhanced flexibility. International Journal of Physical Distribution \& Logistics Management, 39(5), 404-427.

Spekman, R. \& Cousins, P. (2003). Strategic Supply and the Management of Inter- and Intra-Organisational Relationships. Journal of Purchasing and Supply Management, 9(1), 19-29.

Sridharan, V. \& Riley, J. \& Klein, R. \& Miller, J. (2016). How internal integration, information sharing, and training affect supply chain risk management capabilities. International Journal of Physical Distribution \& Logistics Management, 46(10), 953-980.

Talib, F. \& Zillur, R. (2011). Integrating total quality management and supply chain management: similarities and benefits. Journal of Information Technology and Economic, 7(4), 53-85.

Tomlin, B. \& Dong, L. (2012). Managing Disruption Risk: The Interplay Between Operations and Insurance. Management Science, 58(10), 1898-1915.

Tomlin, B. (2006). On the Value of Mitigation and Contingency Strategies for Managing Supply Chain Disruption Risks. Management Science, 52(5), 639-657.
Trkman, P. \& Valadares, M. \& McCormack, K. (2016). Gestión de riesgos de la cadena de suministro orientada al valor: obtiene lo que espera. Industrial Management \& Data Systems, 116(5), 1061-1083.

Tummala, R. \& Schoenherr, T. (2011). Assessing and managing risks using the Supply Chain Risk Management Process. Supply Chain Management: An International Journal, 16(6), 474-483.

Uta, J. (2005). Supply chain risk management: Understanding the business requirements from a practitioner perspective. An International Journal, 16(1), 120-141.

Vilko, J. \& Ritala, P., \& Edelmann, J. (2014). On uncertainty in supply chain risk management. The International Journal of Logistics Management, 25(1), 3-19.

Viswanath, S. \& Srikantha, T. \& Rajendran, P. (2018). Strategic action grids: a study on supply chain risk management in manufacturing industries in India. Benchmarking: An International Journal, 25(8), 3045-3061.

Wieland, A. \& Wallenburg, C. (2012). Dealing with supply chain risks: Linking risk management practices and strategies to performance. International Journal of Physical Distribution \& Logistics Management, 42(10), 887-905.

Wiwin, W. \& Putu, D. \& Udisubakti, C. (2015). Un estudio de caso en una empresa de fabricación de Indonesia. Journal of Manufacturing Technology Management, 4(1), 282-290.

Zerón, M. \& Mendoza, G. \& Quevedo J. (2013). Ventaja competitiva de la cadena de suministros: alianzas, asimetrías organizativas y conflictos. Revista Nacional de Administración, 4(1), 109-118.

Zhu, Q. \& Krikke, H. \& Caniëls, M. (2017). Integrated supply chain risk management: a systematic review. The International Journal of Logistics Management, 28(4), 1123-1141. 